Canadian

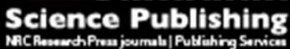

Applied Physiology, Nutrition, and Metabolism Physiologie appliquée, nutrition et métabolisme

\title{
Supplementation with a Proprietary Blend of Ancient Peat and Apple Extract May Improve Body Composition without Affecting Hematology in Resistance-Trained Men
}

\begin{tabular}{|r|l|}
\hline Journal: & Applied Physiology, Nutrition, and Metabolism \\
\hline Manuscript ID & apnm-2015-0241.R1 \\
\hline Manuscript Type: & Article \\
\hline Date Submitted by the Author: & 23-Jun-2015 \\
\hline Complete List of Authors: & $\begin{array}{l}\text { Joy, Jordan; MusclePharm Sports Science Institute, } \\
\text { Falcone, Paul; MusclePharm Sports Science Institute, } \\
\text { Vogel, Roxanne; MusclePharm Sports Science Institute, } \\
\text { Mosman, Matt; MusclePharm Sports Science Institute, } \\
\text { Kim, Michael; MusclePharm Sports Science Institute, } \\
\text { Moon, Jordan; MusclePharm Sports Science Institute, ; United States } \\
\text { Sports Academy, Sports Exercise Science }\end{array}$ \\
\hline Keyword: & $\begin{array}{l}\text { ergogenic aids < athlete performance, body composition, resistance } \\
\text { exercise < exercise, muscle adaptation < muscle, ergogenic aids < } \\
\text { nutrition }\end{array}$ \\
\hline
\end{tabular}


1 Supplementation with a Proprietary Blend of Ancient Peat and Apple Extract May Improve Body

2 Composition without Affecting Hematology in Resistance-Trained Men

3 Jordan M. Joy ${ }^{1}$, Paul H. Falcone ${ }^{1}$, Roxanne M. Vogel ${ }^{1}$, Matt M. Mosman ${ }^{1}$, Michael P. Kim ${ }^{1}$, and Jordan R.

$4 \operatorname{Moon}^{1,2 *}$

5

$6{ }^{1}$ MusclePharm Sports Science Institute, MusclePharm Corp., Denver, CO, USA

$7 \quad{ }^{2}$ Department of Sports Exercise Science, United States Sports Academy, Daphne, AL, USA

8

$9 \quad *$ Corresponding Author:

10 Jordan R. Moon

114721 Ironton St.

12 Building $\mathrm{A}$

13 Denver, CO 80239

$14 \quad P:(616) 901-8698$

15 F: (800)-490-7165

16 jordan@musclepharm.com

17 
1

2

3

4

5

6

7

8

\section{Abstract}

Adenosine $5^{\prime}$-triphosphate (ATP) is primarily known as a cellular source of energy. Increased ATP levels may have the potential to enhance body composition. A novel, proprietary blend of ancient peat and apple extracts, has been reported to increase ATP levels, potentially by enhancing mitochondrial ATP production. Therefore, the purpose of this investigation was to determine the supplement's effects on body composition when consumed during 12 weeks of resistance training. Twenty-five healthy, resistance-trained, male subjects $(27.7 \pm 4.8 \mathrm{y} ; 176.0 \pm 6.5 \mathrm{~cm} ; 83.2 \pm 12.1 \mathrm{~kg})$ completed this study. Subjects supplemented once daily with either 1 serving $(150 \mathrm{mg})$ of a proprietary blend of ancient peat and apple extracts (TRT) or placebo (PLA). Supervised resistance training consisted of 8 weeks of daily undulating periodized training followed by a 2-week overreach and a 2-week taper phase. Body composition was assessed using DEXA and ultrasound at weeks $0,4,8,10$, and 12 . Vital signs and blood markers were assessed at weeks 0,8 , and 12 . Significant group $x$ time $(p<0.05)$ interactions were present for ultrasound determined cross sectional area, which increased in TRT $\left(+0.91 \mathrm{~cm}^{2}\right)$ versus PLA $\left(-0.08 \mathrm{~cm}^{2}\right)$, as well as muscle thickness (TRT: +0.46 ; PLA: $+0.04 \mathrm{~cm})$. A significant group $x$ time $(p<0.05)$ interaction existed for creatinine (TRT: +0.06; PLA: $+0.15 \mathrm{mg} / \mathrm{dL}$ ), triglycerides (TRT: +24.1; PLA: $-20.2 \mathrm{mg} / \mathrm{dL}$ ), and VLDL (TRT: +4.9; PLA: -3.9mg/dL), which remained within clinical ranges. Supplementation with a proprietary blend of ancient peat and apple extracts may enhance resistance training induced skeletal muscle hypertrophy without affecting fat mass or blood chemistry in healthy males.

Keywords: ElevATP, strength training, weight lifting, muscle mass, fat mass, blood chemistry, safety, periodization, sports nutrition, sport 


\section{1}

2

3

4

5

6

8

\section{Introduction}

Adenosine-5' -triphosphate (ATP) and ATP metabolites are involved in a myriad of biological processes

including cardiac function, neurotransmission, blood flow, and muscle contraction (Agteresch et al.

1999; Kushmerick and Conley 2002), and it is strongly suggested that increased ATP levels correlate with improved health and performance (Jordan et al. 2004; Herda et al. 2008; Swamy et al. 2011). Direct supplementation with exogenous ATP has produced mixed results in terms of increasing ATP when measured in whole blood (Jordan et al. 2004; Arts et al. 2012; Burnstock et al. 2012). Therefore, an indirect approach for increasing endogenous ATP levels may be desirable. Previously, oral supplementation with a proprietary blend of ancient peat and apple extracts has been demonstrated to increase intracellular ATP levels in whole blood and muscle, suggesting increased activity of bodily processes that produce or release endogenous ATP (Reyes-Izquierdo et al. 2013; Reyes-Izquierdo et al. 2014).

Reyes-Izquierdo and colleagues (Reyes-Izquierdo et al. 2014) determined that a 150mg dose of this blend of ancient peat and apple extracts significantly increased ATP in cellular fraction of blood compared to placebo. Specifically, blood ATP increased by $40 \% 60$ minutes following ingestion and dropped to $28 \% 120$ minutes following ingestion. A muscle biopsy was conducted in one resting subject, and ATP levels in muscle tissue were observed to increase $281 \%$ at 60 minutes and $433 \%$ at 120 minutes following ingestion (Reyes-Izquierdo et al. 2014). Preliminary reports from this laboratory suggest this occurs without increases in reactive oxygen species, which may be associated with increased ATP production (Chang et al. 2010). In fact, ancient peat and apple extracts may actually decrease reactive oxygen species (Reyes-Izquierdo et al. 2013), possibly blunting the increase in reactive oxygen species caused by resistance training (Alessio et al. 2000). 
1 Despite these observations, supplementation for indirect ATP enhancement is yet to be evaluated for

2 potential to induce body composition changes in response to resistance training. However, the existing

3 data on a blend of ancient peat and apple extracts for increasing both whole blood and muscle ATP

4 levels (Reyes-Izquierdo et al. 2013; Reyes-Izquierdo et al. 2014) support the plausibility that chronic

5 supplementation may yield changes in body composition. Additionally, because this is a novel

6 ingredient, chronic supplementation must be verified as safe. Therefore, the purpose of this study is to

7 determine the effects of a proprietary blend of ancient peat and apple extracts on body composition

8 and hematological safety markers. It was hypothesized that the supplement will increase lean muscle

9 mass and muscle hypertrophy without producing a change in body fat or hematological safety markers.

10

11

12 Twenty-five healthy, resistance-trained, male subjects $(27.7 \pm 4.8 \mathrm{y} ; 176.0 \pm 6.5 \mathrm{~cm} ; 83.2 \pm 12.1 \mathrm{~kg})$

13

14

15

16

17

18

19

20 21 study.

22

\section{Methods}

Participants

completed this study. Thirty-three subjects were recruited, and three subjects did not complete the study due to scheduling conflicts, three were not compliant with protocols, and two sustained injuries during the study. Each subject was required to be capable of lifting $1.5 x$ their bodyweight in the squat and deadlift and $1 x$ bodyweight in the bench press. At baseline, the placebo (PLA) group was able to squat $1.71 \pm 0.21$, bench press $1.45 \pm 0.19$, and deadlift $2.17 \pm 0.25$ times their bodyweight, and the treatment (TRT) group was able to squat $1.66 \pm 0.24$, bench press $1.31 \pm 0.20$, and deadlift $1.93 \pm 0.27$ times their bodyweight. Approval for research with human subjects was obtained from a registered IRB, and subjects were provided with written informed consent documents prior to participation in the

\section{Experimental Design}


1 Subjects were randomly assigned to either the PLA $(n=11)$ or TRT $(n=14)$ groups. They were instructed

2 to consume 1 serving of either PLA or TRT (elevATP ${ }^{\circledR}$, VDF FutureCeuticals Inc., Momence, IL; 150mg) 45

3 minutes prior to training on training days or at a similar time of day on rest days (for detailed

4 supplement composition, see (Reyes-Izquierdo et al. 2013)). Supplement vials were weighed to ensure

5 compliance. Both subjects and researchers were blinded to the PLA and TRT groups. The researchers

6 received two sets of supplements labeled A and B from a third-party manufacturer. The blind was

7 broken only after the completion of data analysis. Subjects were resistance trained under the guidance

8 of a certified strength and conditioning specialist 3 days per week for 8 weeks followed by a 2-week

9 overreach and 2-week taper phase corresponding to weeks 9-10 and 11-12, respectively, in a design similar to that previously described (Wilson et al. 2013). A eucaloric diet consisting of 50\% calories from

11 carbohydrates, $25 \%$ from protein, and $25 \%$ from fat was prescribed to all subjects at the onset of the

12 study, and diets were tracked weekly via 3-day food logs. Total calories were determined for each

13 individual based on the Mifflin St. Jeor equation adjusted for activity level. Subjects were measured at

14 weeks $0,4,8,10$, and 12 for all body composition variables. Blood draws and vital signs measurements

15 were conducted at weeks 0,8 , and 12 . Body composition variables collected consisted of DEXA-

16 determined lean soft tissue (LST), fat mass (FM), and body fat percentage (BF\%), ultrasound-determined

17 cross-sectional area (CSA), muscle thickness (MT), and fat thickness (FT).

Resistance Training Program

Weeks 1-8 consisted of one muscle hypertrophy oriented workout consisting of barbell back squat,

20 bench press, deadlift, incline bench press, power squat, hammer strength isolateral bench press, leg

21 press, leg extension, leg curl, and triceps extension performed for 3 sets of 6-12 repetitions at $65-80 \%$

22 1RM intensity, one power day consisting of barbell back squat, bench press, and deadlift performed for

235 sets of $2-5$ repetitions at $40-60 \% 1 \mathrm{RM}$ intensity with a goal of high velocity of movement, and one

24 strength oriented day consisting of barbell back squat, bench press, deadlift, shoulder press, and 
1 pulldown performed for 3 sets of 1-5 repetitions at $85-100 \% 1 \mathrm{RM}$ intensity. After performing the main

2 exercises on the power day, subjects performed bent over row, pulldown, dumbbell row, shoulder

3 press, lateral raise, and bicep curl exercises for the goal of muscle hypertrophy as described for chest

4 and leg exercises. Following the resistance exercises on the strength day, participants performed 2-6

5 sets of 10-30s Wingates with 2-4 minutes rest on a cycle ergometer. Participants rested 48-72 hours

6 between each training day, and 30-120s between sets on the hypertrophy day or 2-5min between sets

7 on the power and strength days. During the overreach phase, participants performed high volume

8 workouts, similar to the hypertrophy oriented workouts performed during weeks 1-8, on Monday

9 through Thursday with a strength-oriented workout or performance testing conducted on Friday for

weeks 9 and 10, respectively. The taper weeks consisted of one power day on Mondays then strength

11 and power day on both Wednesdays and Fridays performed at low volume for back squat, bench press,

12 and deadlift only. Each training cycle began with the lowest intensity of the range provided, and

13 intensity progressively increased as repetitions decreased and rest time increased from session to

14 session.

15

16

17

18

\section{Measurements}

Urine specific gravity was determined on each body composition testing day to ensure measurements were conducted in a euhydrated state. Adequate hydration was considered to be a USG of $1.000-$ 1.030. On 3 occasions, a participant was required to drink water until another urine sample could be submitted and verified for adequate hydration status. Body weight was determined using a calibrated column scale (SECA, Chino, CA). Body composition was analyzed for whole-body LST, FM, and BF\% as well as segmental LST using DEXA (Lunar Prodigy Primo, General Electric, Fairfield, CN) using enCORE software (Version 15, Madison, WN). Test-retest reliability for DEXA LST, BF\%, and FM, as measured using 15 subjects, resulted in an average ICC of >0.99. CSA, MT, and FT were determined using ultrasound (Logiq e, General Electric, Fairfield, CN). Ultrasonography determined CSA was measured at 
$175 \%$ femur length, as defined as the distance from the anterior superior iliac spine to the superior aspect

2 of the patella. MT of the quadriceps was measured at $50 \%$ femur length, defined as the distance from

3 the greater trochanter of the femur to the lateral epicondyle of the femur. MT was defined as the

4 combined thickness of the vastus lateralis and vastus intermedius. The distance from the superficial

5 aspect the femur to the deep aspect of the superficial fascia of the vastus lateralis was measured. FT

6 was measured at the same site as MT, and it was defined as the distance from the superficial aspect of

7 the vastus lateralis fascial layer to the deep aspect of the hypodermis. Test-retest reliability for

8 ultrasound measurements, as determined using 5 subjects, resulted in an average ICC $>0.99$. Vital signs

9 were determined using an automated, digital sphygmomanometer (Omron Corporation, Kyoto, Japan).

Blood draws were performed via venipuncture by a trained phlebotomist. Following a 10-hour fast, all

11 subjects submitted a blood sample for analysis in the morning to control for diurnal variations. Blood

12 variables consisted of white blood cell count (WBC), red blood cell count (RBC), hemoglobin, hematocrit,

mean corpuscular volume (MCV), mean corpuscular hemoglobin $(\mathrm{MCH})$, mean corpuscular hemoglobin

concentration (MCHC), red blood cell distribution width (RDW), platelets (absolute), neutrophils

15 (percent and absolute), lymphocytes (percent and absolute), monocytes (percent and absolute),

eosinophils (percent and absolute), basophils (percent and absolute), serum glucose, blood urea

17 nitrogen (BUN), creatinine, estimated glomerular filtration rate (eGFR), BUN:creatinine, sodium,

18 potassium, chloride, carbon dioxide, calcium, protein, albumin, globulin, albumin:globulin (A/G),

19 bilirubin, alkaline phosphatase, aspartate aminotransferase (AST), alanine aminotransferase (ALT), total

cholesterol, triglycerides, high density lipoprotein (HDL) cholesterol, very low density lipoprotein (VLDL),

21 and low density lipoprotein (LDL) cholesterol. Blood variables were analyzed by a third party (Laboratory

22 Corporation of America, Denver, CO). Inter-test reliability results from 12 men and women measured up

23 to one week apart at the aforementioned laboratory resulted in no significant differences from day-to-

24 day $(p>0.05)$ and an average inter-test Coefficient of Variation of $6.9 \%$ for all tests. 


\section{Statistical Analyses}

A repeated measures ANOVA was performed to assess group, time, and group $\mathrm{x}$ time interactions with a significant $p$-value considered as $\leq 0.05$. Bonferroni post-hoc analyses were used to locate differences following a significant group $x$ time interaction. Dependent T-test were conducted to determine withingroup time effects for all body composition variables and hematology variables with a significant interaction. On two occasions, a dependent variable was significantly different between groups at baseline, and ANCOVAs were performed to assess group, time, and group x time interactions. Independent T-tests were conducted on the delta values for each time point. Statistica (Version 10, Statsoft, Tulsa, OK) was used for all statistical analyses.

\section{Results}

A significant main effect for time and a group $x$ time $(p<0.05)$ interaction (observed power $>0.99)$ was present for CSA. CSA was significantly increased in TRT versus PLA at weeks 8, 10, and 12 (Table 1). Moreover, CSA increased in TRT compared to PLA between all time points except for between weeks 0 and 4 using independent T-tests $(p<0.05$; Figure 1). There was a significant main effect for time and a group $x$ time $(p<0.05)$ interaction (observed power $=0.92)$ observed for MT (Figure 2, Table 1). Wherein, MT increased to a greater extent in TRT than PLA from pre to weeks 10 and 12. A significant main effect for time $(p<0.05)$ was found for body weight, FT, LST, FM, BF\%, and LST of the arms, legs, and trunk, but no significant group $\mathrm{x}$ time interactions existed for these variables (Table 1).

No changes were observed for systolic or diastolic blood pressure or heart rate. A significant group $\mathrm{x}$ time $(p<0.05)$ interaction (observed power $=0.89)$ was present for creatinine, which increased from pre to post in both TRT and PLA (TRT: +0.06 ; PLA: $+0.15 \mathrm{mg} / \mathrm{dL})$. There was a significant group $\mathrm{x}$ time $(\mathrm{p}<$ 0.05 ) effect (observed power $=0.89$ ) for triglycerides from pre to post (TRT: $+24.1 ;$ PLA: $-20.2 \mathrm{mg} / \mathrm{dL}$ ). Finally, a significant group $x$ time $(p<0.05)$ interaction (observed power $=0.89$ ) existed for VLDL from 
1 pre to post (TRT: +4.9; PLA: $-3.9 \mathrm{mg} / \mathrm{dL}$ ). However, differences were present for triglycerides and VLDL at

2 baseline. Further analysis using ANCOVA revealed no significant difference for triglycerides $(p=0.09)$ or

$3 \operatorname{VLDL}(p=0.11)$. No other significant interactions were observed for any safety markers, and each

4 marker remained within the physiological reference range (Tables 2, 3, and 4).

\section{Discussion}

6 The results of this study support the hypotheses. Although no interactions were observed for any

7 measurement of LST between groups, both CSA and MT increased while measures of body fat content

8 and body weight were unchanged. In the PLA group, CSA tended to decline following the overreach and

9 taper phases, while TRT increased. We hypothesize that the PLA group was unable to adapt to the

10 overreach stimulus, whereas the supplement aided this adaptation. Moreover, no abnormal changes in

11 vital signs or blood markers were detected. Creatinine increased to a greater extent in PLA compared to

12 TRT, and PLA had elevated triglycerides and VLDL at baseline versus TRT, which appeared to normalize

13 throughout the trial. Furthermore, each blood marker remained within the clinical reference range.

14 With these considerations, it is unlikely that these changes were produced by supplementation.

15 Previous reports on direct ATP supplementation and resistance training are in agreement with the

16 present results. Wherein, muscle mass increased without changes in fat mass (Wilson et al. 2013).

17 Although it has also been reported that 12 weeks of direct ATP supplementation is safe (Wilson et al.

18 2013), and Coolen and colleagues (Coolen et al. 2011) observed no changes in blood markers following 4

19 weeks direct supplementation with $5 \mathrm{~g} /$ day of ATP, it may not be appropriate to compare direct and

20 indirect ATP enhancement, due to their dissimilar compositions and dosage levels, and because their

21 respective mechanisms of action are likely different.

22 The blend of ancient peat and apple extracts may be capable of promoting skeletal muscle hypertrophy

23 by increasing whole-blood ATP levels (Reyes-Izquierdo et al. 2013; Reyes-Izquierdo et al. 2014) with a 
1 subsequent augmentation of blood flow. ATP and adenosine have been known to induce vasodilation

2 following release from the erythrocytes via production of nitric oxide and prostacyclin (Nyberg et al.

3 2010; Sprague et al. 2011), and it has been recently demonstrated that exogenous ATP supplementation

4 is capable of increasing exercise-induced blood flow (Jager et al. 2014). Improved blood flow may

5 increase nutrient delivery. Thus, there is a possibility for a greater effect of circulating amino acids

6 (Morgan et al. 1971; Bohe et al. 2003), glucose (Baron et al. 1994; Baron and Clark 1997), and oxygen

7 (Buchheit et al. 2009), which may enhance anabolic signaling and/or acute exercise performance,

8 leading to amplified chronic adaptations (Buchheit et al. 2009; Rodriguez et al. 2009).

9 In addition to increasing whole blood ATP levels, a proposed mechanism of action for ancient peat and 10 apple extracts is intracellular ATP production (Reyes-Izquierdo et al. 2013; Reyes-Izquierdo et al. 2014).

11 With greater ATP levels, it can be speculated that cyclic adenosine monophosphate induced inhibition of

12 the mammalian target of rapamycin may be attenuated, thereby permitting enhanced anabolic signaling

13 and possibly muscle protein synthesis (Xie et al. 2011). An improved resistance to fatiguing exercise is

14 also possible via this mechanism, such as with previous research on creatine monohydrate, which

15 increases ATP availability (Racette 2003), and previous research regarding creatine's capabilities for

16 increasing muscle mass have been well established (Buford et al. 2007). However, more thorough

17 research is required prior to considering this as a viable mechanism, due to the fact that previously

18 enhanced intramuscular ATP levels have only been examined in one resting subject (Reyes-Izquierdo et

al. 2014). However, the blend of ancient peat and apple extracts may be advantageous to direct ATP

20 supplementation for these reasons, as direct ATP supplementation may only exert extracellular effects

21 due to rapid degradation to its metabolites (Hochachka et al. 1991; Gorman et al. 2007; Mortensen et al.

22 2011; Jager et al. 2014). The present study was limited by not measuring ATP levels either in whole-

23 blood or muscle tissue. Thus, it cannot be determined if the effects reported by Reyes-lzquierdo et al.

24 (Reyes-Izquierdo et al. 2013; Reyes-Izquierdo et al. 2014) persist in a chronic setting, as the 
1 supplement's effects may be attenuated with daily administration. A second limitation of the study

2 includes the lack of a non-exercising control group.

3 This is the first study examining the effects of indirect ATP enhancement with supplementation on body

4 composition. Daily supplementation with a proprietary blend of ancient peat and apple extracts may be

5 beneficial for enhancing the hypertrophic effects of resistance training on skeletal muscle without

6 augmenting body fat content or safety parameters. Therefore, strength athletes, bodybuilders, and

7 contact sport athletes may have an interest using this supplement. It is possible that this effect is

8 exerted via increased blood flow, anabolic signaling, and/or enhanced energy status. However, future

9 research is necessary to elaborate on the mechanisms of the present observations, and possibly in a

10 younger versus older population. Future research may also be interested in determining the combined

11 effects of a blend of ancient peat and apple extracts with creatine and/or nitrate, as both are thought to

12 affect ATP availability.

\section{Acknowledgements}

14 The authors would like to thank the participants and VDF FutureCeuticals Inc. for funding this 15 investigation. 


\section{References}

Agteresch, H.J., Dagnelie, P.C., van den Berg, J.W., and Wilson, J.H. 1999. Adenosine triphosphate: established and potential clinical applications. Drugs 58: 211-32.

Alessio, H.M., Hagerman, A.E., Fulkerson, B.K., Ambrose, J., Rice, R.E., and Wiley, R.L. 2000. Generation of reactive oxygen species after exhaustive aerobic and isometric exercise. Med Sci Sports Exerc 32: 1576-81.

Arts, I.C., Coolen, E.J., Bours, M.J., Huyghebaert, N., Stuart, M.A., Bast, A., and Dagnelie, P.C. 2012. Adenosine 5'-triphosphate (ATP) supplements are not orally bioavailable: a randomized, placebocontrolled cross-over trial in healthy humans. J Int Soc Sports Nutr 9: 16.

Baron, A.D. and Clark, M.G. 1997. Role of blood flow in the regulation of muscle glucose uptake. Annu Rev Nutr 17: 487-99.

Baron, A.D., Steinberg, H., Brechtel, G., and Johnson, A. 1994. Skeletal muscle blood flow independently modulates insulin-mediated glucose uptake. Am J Physiol 266: E248-53.

Bohe, J., Low, A., Wolfe, R.R., and Rennie, M.J. 2003. Human muscle protein synthesis is modulated by extracellular, not intramuscular amino acid availability: a dose-response study. J Physiol 552: 315-24. Buchheit, M., Cormie, P., Abbiss, C.R., Ahmaidi, S., Nosaka, K.K., and Laursen, P.B. 2009. Muscle deoxygenation during repeated sprint running: Effect of active vs. passive recovery. Int J Sports Med 30: 418-25.

Buford, T.W., Kreider, R.B., Stout, J.R., Greenwood, M., Campbell, B., Spano, M., Ziegenfuss, T., Lopez, H., Landis, J., and Antonio, J. 2007. International Society of Sports Nutrition position stand: creatine supplementation and exercise. J Int Soc Sports Nutr 4: 6.

Burnstock, G., Knight, G.E., and Greig, A.V. 2012. Purinergic signaling in healthy and diseased skin. J Invest Dermatol 132: 526-46.

Chang, J.C., Kou, S.J., Lin, W.T., and Liu, C.S. 2010. Regulatory role of mitochondria in oxidative stress and atherosclerosis. World J Cardiol 2: 150-9.

Coolen, E.J., Arts, I.C., Bekers, O., Vervaet, C., Bast, A., and Dagnelie, P.C. 2011. Oral bioavailability of ATP after prolonged administration. Br J Nutr 105: 357-66.

Gorman, M.W., Feigl, E.O., and Buffington, C.W. 2007. Human plasma ATP concentration. Clin Chem 53: 318-25.

Herda, T.J., Ryan, E.D., Stout, J.R., and Cramer, J.T. 2008. Effects of a supplement designed to increase ATP levels on muscle strength, power output, and endurance. J Int Soc Sports Nutr 5: 3. Hochachka, P.W., Bianconcini, M.S., Parkhouse, W.S., and Dobson, G.P. 1991. On the role of actomyosin ATPases in regulation of ATP turnover rates during intense exercise. Proc Natl Acad Sci U S A 88: 5764-8. Jager, R., Roberts, M.D., Lowery, R.P., Joy, J.M., Cruthirds, C.L., Lockwood, C.M., Rathmacher, J.A., Purpura, M., and Wilson, J.M. 2014. Oral adenosine-5'-triphosphate (ATP) administration increases blood flow following exercise in animals and humans. J Int Soc Sports Nutr 11: 28.

Jordan, A.N., Jurca, R., Abraham, E.H., Salikhova, A., Mann, J.K., Morss, G.M., Church, T.S., Lucia, A., and Earnest, C.P. 2004. Effects of oral ATP supplementation on anaerobic power and muscular strength. Med Sci Sports Exerc 36: 983-90.

Kushmerick, M.J. and Conley, K.E. 2002. Energetics of muscle contraction: the whole is less than the sum of its parts. Biochem Soc Trans 30: 227-31.

Morgan, H.E., Earl, D.C., Broadus, A., Wolpert, E.B., Giger, K.E., and Jefferson, L.S. 1971. Regulation of protein synthesis in heart muscle. I. Effect of amino acid levels on protein synthesis. J Biol Chem 246: 2152-62. 
Mortensen, S.P., Thaning, P., Nyberg, M., Saltin, B., and Hellsten, Y. 2011. Local release of ATP into the arterial inflow and venous drainage of human skeletal muscle: insight from ATP determination with the intravascular microdialysis technique. J Physiol 589: 1847-57. Nyberg, M., Mortensen, S.P., Thaning, P., Saltin, B., and Hellsten, Y. 2010. Interstitial and plasma adenosine stimulate nitric oxide and prostacyclin formation in human skeletal muscle. Hypertension 56 : 1102-8.

Racette, S.B. 2003. Creatine supplementation and athletic performance. Journal of Orthopaedic \& Sports Physical Therapy 33: 615-621.

Reyes-Izquierdo, T., Nemzer, B., Argumedo, R., Shu, C., Huynh, L., and Pietrzkowski, Z. 2013. Effect of the dietary supplement ElevATP on blood ATP level: An acute pilot clinical study. J Aging Res Clin Practice 2: 178-184.

Reyes-Izquierdo, T., Shu, C., Argumedo, R., Nemzer, B., and Pietrzkowski, Z. 2014. The effect of elevATP'м on whole blood ATP levels: a single dose, crossover clinical study. J. Aging Res. Clin. Practice 3: 56-60. Rodriguez, N.R., DiMarco, N.M., and Langley, S. 2009. Nutrition and athletic performance. Medicine and science in sports and exercise 41: 709-731.

Sprague, R.S., Bowles, E.A., Achilleus, D., and Ellsworth, M.L. 2011. Erythrocytes as controllers of perfusion distribution in the microvasculature of skeletal muscle. Acta Physiol (Oxf) 202: 285-92. Swamy, M.S., Sivanna, N., Tamatam, A., and Khanum, F. 2011. Effect of poly phenols in enhancing the swimming capacity of rats. Functional Foods in Health and Disease 1: 482-491.

Wilson, J.M., Joy, J.M., Lowery, R.P., Roberts, M.D., Lockwood, C.M., Manninen, A.H., Fuller, J.C., De Souza, E.O., Baier, S.M., Wilson, S.M., and Rathmacher, J.A. 2013. Effects of oral adenosine-5'triphosphate supplementation on athletic performance, skeletal muscle hypertrophy and recovery in resistance-trained men. Nutr Metab (Lond) 10: 57.

Xie, J., Ponuwei, G.A., Moore, C.E., Willars, G.B., Tee, A.R., and Herbert, T.P. 2011. cAMP inhibits mammalian target of rapamycin complex-1 and-2 (mTORC1 and 2) by promoting complex dissociation and inhibiting mTOR kinase activity. Cellular signalling 23: 1927-1935. 
Table 1. Body Composition Data.

\begin{tabular}{|c|c|c|c|c|c|c|c|}
\hline Variable & Group & Pre & Week 4 & Week 8 & Week 10 & Post & $\mathrm{p}$ \\
\hline \multirow[t]{2}{*}{$\operatorname{CSA}\left(\mathrm{cm}^{2}\right)$} & PLA & $3.60 \pm 1.57$ & $3.85 \pm 1.49^{\mathrm{a}}$ & $3.73 \pm 1.32$ & $3.61 \pm 1.27$ & $3.52 \pm 1.39^{\mathrm{bc}}$ & \multirow{2}{*}{$<0.001$} \\
\hline & TRT & $4.07 \pm 1.52$ & $4.24 \pm 1.4^{\mathrm{a}}$ & $4.52 \pm 1.41 * \mathrm{ab}$ & $4.66 \pm 1.50 * a b c$ & $4.99 \pm 1.63 *$ abcd & \\
\hline \multirow[t]{2}{*}{$\mathrm{MT}(\mathrm{cm})$} & PLA & $5.50 \pm 0.72$ & $5.47 \pm 0.50$ & $5.53 \pm 0.74$ & $5.43 \pm 0.66^{c}$ & $5.55 \pm 0.68^{d}$ & \multirow{2}{*}{0.001} \\
\hline & TRT & $5.25 \pm 0.73$ & $5.58 \pm 0.63^{\mathrm{a}}$ & $5.58 \pm 0.68^{\mathrm{a}}$ & $5.62 \pm 0.65^{* a}$ & $5.71 \pm 0.68 * \mathrm{abcd}$ & \\
\hline \multirow[t]{2}{*}{$\mathrm{FT}(\mathrm{cm})$} & PLA & $0.52 \pm 0.20$ & $0.49 \pm 0.19$ & $0.53 \pm 0.19$ & $0.48 \pm 0.18$ & $0.53 \pm 0.16$ & \multirow{2}{*}{0.83} \\
\hline & TRT & $0.40 \pm 0.39$ & $0.43 \pm 0.45$ & $0.46 \pm 0.47$ & $0.39 \pm 0.33$ & $0.45 \pm 0.41$ & \\
\hline \multirow[t]{2}{*}{ LST (kg) } & PLA & $65.3 \pm 8.1$ & $65.2 \pm 6.5$ & $65.7 \pm 6.6$ & $66.2 \pm 6.8$ & $65.5 \pm 6.9$ & \multirow{2}{*}{0.75} \\
\hline & TRT & $67.4 \pm 6.2$ & $68.0 \pm 6.2$ & $68.4 \pm 6.0$ & $69.3 \pm 6.2$ & $67.8 \pm 5.4$ & \\
\hline \multirow[t]{2}{*}{ FM (kg) } & PLA & $16.7 \pm 5.6$ & $18.6 \pm 6.3$ & $18.4 \pm 6.6$ & $18.5 \pm 6.9$ & $18.5 \pm 6.5$ & \multirow{2}{*}{0.47} \\
\hline & TRT & $13.4 \pm 10.3$ & $14.2 \pm 9.8$ & $14.5 \pm 9.6$ & $14.6 \pm 10.1$ & $15.1 \pm 9.8$ & \\
\hline \multirow[t]{2}{*}{$\mathrm{BF} \%$} & PLA & $20.1 \pm 5.4$ & $21.8 \pm 5.5$ & $21.4 \pm 5.8$ & $21.3 \pm 5.9$ & $21.6 \pm 5.7$ & \multirow{2}{*}{0.37} \\
\hline & TRT & $15.5 \pm 8.0$ & $16.3 \pm 7.5$ & $16.5 \pm 7.4$ & $16.3 \pm 7.4$ & $17.2 \pm 7.7$ & \\
\hline \multirow[t]{2}{*}{ Body Weight (kg) } & PLA & $83.7 \pm 10.5$ & $86.3 \pm 10.6$ & $86.1 \pm 11.5$ & $87.3 \pm 12.1$ & $86.4 \pm 11.7$ & \multirow{2}{*}{1.00} \\
\hline & TRT & $82.8 \pm 13.6$ & $85.2 \pm 13.6$ & $85.2 \pm 14.0$ & $85.9 \pm 13.8$ & $85.3 \pm 13.5$ & \\
\hline \multirow[t]{2}{*}{ R Leg LST (kg) } & PLA & $10.6 \pm 1.7$ & $10.9 \pm 1.9$ & $10.9 \pm 1.5$ & $11.2 \pm 1.7$ & $10.9 \pm 1.3$ & \multirow{2}{*}{0.39} \\
\hline & TRT & $11.3 \pm 1.1$ & $11.5 \pm 1.2$ & $11.7 \pm 1.1$ & $11.6 \pm 1.2$ & $11.5 \pm 1.1$ & \\
\hline \multirow[t]{2}{*}{ L Leg LST (kg) } & PLA & $10.5 \pm 1.5$ & $10.8 \pm 1.7$ & $10.8 \pm 1.4$ & $11.1 \pm 1.6$ & $10.7 \pm 1.1$ & \multirow{2}{*}{0.35} \\
\hline & TRT & $11.1 \pm 1.1$ & $11.3 \pm 1.2$ & $11.4 \pm 1.1$ & $11.4 \pm 1.0$ & $11.3 \pm 0.9$ & \\
\hline \multirow[t]{2}{*}{ R Arm LST (kg) } & PLA & $4.7 \pm 0.6$ & $4.5 \pm 0.5$ & $4.3 \pm 0.5$ & $4.2 \pm 0.5$ & $4.1 \pm 0.5$ & \multirow{2}{*}{0.84} \\
\hline & TRT & $4.8 \pm 0.7$ & $4.6 \pm 0.7$ & $4.3 \pm 0.5$ & $4.4 \pm 0.6$ & $4.2 \pm 0.7$ & \\
\hline \multirow[t]{2}{*}{ L Arm LST (kg) } & PLA & $4.5 \pm 0.5$ & $4.5 \pm 0.4$ & $4.1 \pm 0.5$ & $4.0 \pm 0.4$ & $4.0 \pm 0.5$ & \multirow{2}{*}{0.61} \\
\hline & TRT & $4.5 \pm 0.7$ & $4.4 \pm 0.7$ & $4.0 \pm 0.5$ & $4.1 \pm 0.6$ & $4.1 \pm 0.7$ & \\
\hline \multirow[t]{2}{*}{ Trunk LST (kg) } & PLA & $30.8 \pm 4.3$ & $30.2 \pm 2.9$ & $31.2 \pm 3.4$ & $31.5 \pm 3.5$ & $31.6 \pm 4.6$ & \multirow{2}{*}{0.27} \\
\hline & TRT & $31.3 \pm 3.2$ & $31.8 \pm 3.5$ & $32.5 \pm 3.9$ & $33.7 \pm 4.2$ & $30.4 \pm 8.4$ & \\
\hline
\end{tabular}

Data are presented as mean \pm standard deviation. * indicates significantly different from PLA at the

4 interaction. Significant time effects $(p<0.05)$ are indicated by a (different from baseline), b (different 
1 Table 2. Vital Signs and Blood Lipid Data.

\begin{tabular}{|c|c|c|c|c|c|c|}
\hline & & & & & Reference & \\
\hline Variable & Treatment & PRE & Week 8 & POST & Interval & $\mathrm{p}$ \\
\hline \multirow[t]{3}{*}{ Systolic BP (mm Hg) } & PLA & $127 \pm 12.0$ & $126.0 \pm 12.2$ & $127.0 \pm 12.2$ & \multirow{3}{*}{$90-120$} & \multirow{3}{*}{0.98} \\
\hline & & & & & & \\
\hline & TRT & $125.0 \pm 13.3$ & $123.1 \pm 10.6$ & $123.9 \pm 11.1$ & & \\
\hline \multirow[t]{3}{*}{ Diastolic BP (mm Hg) } & PLA & $76.1 \pm 9.4$ & $77.0 \pm 8.0$ & $76.4 \pm 9.4$ & \multirow{3}{*}{$60-80$} & \multirow{3}{*}{0.38} \\
\hline & & & & & & \\
\hline & TRT & $76.7 \pm 10.4$ & $73.1 \pm 7.5$ & $76.1 \pm 5.5$ & & \\
\hline \multirow[t]{3}{*}{ Heart Rate (BPM) } & PLA & $68.9 \pm 10.6$ & $70.1 \pm 10.1$ & $70.0 \pm 7.7$ & \multirow{3}{*}{$<100$} & \multirow{3}{*}{0.79} \\
\hline & & & & & & \\
\hline & TRT & $59.0 \pm 8.2$ & $63.4 \pm 7.3$ & $63.0 \pm 7.0$ & & \\
\hline \multirow[t]{2}{*}{ Total Cholesterol } & PLA & $175.9 \pm 41.9$ & $175.1 \pm 43.2$ & $172.8 \pm 41.0$ & \multirow{3}{*}{$100-199$} & \multirow{3}{*}{0.55} \\
\hline & & & & & & \\
\hline$(\mathrm{mg} / \mathrm{dL})$ & TRT & $162.1 \pm 27.8$ & $160.1 \pm 21.2$ & $157.3 \pm 23.9$ & & \\
\hline \multirow[t]{3}{*}{ Triglycerides $(\mathrm{mg} / \mathrm{dL})$} & PLA & $109.7 \pm 53.0$ & $81.4 \pm 37.9$ & $89.5 \pm 33.3$ & \multirow{3}{*}{$0-149$} & \multirow{3}{*}{$0.09 \ddagger$} \\
\hline & & & & & & \\
\hline & TRT & $68.1 \pm 28.5$ & $67.7 \pm 29.6$ & $92.3 \pm 43.5$ & & \\
\hline \multirow[t]{2}{*}{ High Density } & PLA & $50.1 \pm 13.3$ & $47.6 \pm 12.6$ & $48.5 \pm 11.2$ & \multirow{3}{*}{$>39$} & \multirow{3}{*}{0.63} \\
\hline & & & & & & \\
\hline Lipoprotein (mg/dL) & TRT & $53.5 \pm 13.8$ & $52.1 \pm 15.0$ & $51.0 \pm 16.5$ & & \\
\hline \multirow[t]{2}{*}{ Very Low Density } & PLA & $21.9 \pm 10.5$ & $16.3 \pm 7.6$ & $18.0 \pm 6.8$ & \multirow{3}{*}{$5-40$} & \multirow{3}{*}{$0.11=$} \\
\hline & & & & & & \\
\hline Lipoprotein (mg/dL) & TRT & $13.6 \pm 5.8$ & $13.6 \pm 6.0$ & $18.5 \pm 8.9$ & & \\
\hline \multirow[t]{2}{*}{ Low Density } & PLA & $103.9 \pm 31.7$ & $111.2 \pm 31.2$ & $106.4 \pm 31.0$ & \multirow{3}{*}{$0-99$} & \multirow{3}{*}{0.32} \\
\hline & & & & & & \\
\hline Lipoprotein (mg/dL) & TRT & $94.9 \pm 31.1$ & $94.4 \pm 24.8$ & $87.9 \pm 23.2$ & & \\
\hline \multicolumn{7}{|c|}{ Data are presented as mean \pm standard deviation. The $p$-value is derived from an ANOVA and } \\
\hline \multicolumn{7}{|c|}{ representative of a group by time interaction. $¥$ indicates the $p$-value of an ANCOVA in place of an } \\
\hline
\end{tabular}


Table 3. Hematology Data.

\begin{tabular}{|c|c|c|c|c|c|c|}
\hline Variable & Treatment & PRE & Week 8 & POST & Reference Interval & $p$ \\
\hline \multirow[t]{3}{*}{ WBC (x10E3/uL) } & PLA & $5.7 \pm 1.5$ & $5.7 \pm 1.0$ & $5.7 \pm 1.2$ & \multirow{3}{*}{$3.4-10.8$} & \multirow{3}{*}{0.83} \\
\hline & & & & & & \\
\hline & TRT & $5.8 \pm 1.6$ & $5.9 \pm 1.7$ & $6.0 \pm 1.2$ & & \\
\hline \multirow[t]{3}{*}{ RBC (x10E6/uL) } & PLA & $5.3 \pm 0.3$ & $5.4 \pm 0.4$ & $5.4 \pm 0.3$ & \multirow{3}{*}{$4.14-5.80$} & \multirow{3}{*}{0.22} \\
\hline & & & & & & \\
\hline & TRT & $5.3 \pm 0.3$ & $5.3 \pm 0.3$ & $5.3 \pm 0.2$ & & \\
\hline \multirow[t]{3}{*}{ Hemoglobin $(\mathrm{g} / \mathrm{dL})$} & PLA & $16.2 \pm 1.2$ & $16.4 \pm 1.3$ & $16.4 \pm 1.0$ & \multirow{3}{*}{$12.6-17.7$} & \multirow{3}{*}{0.20} \\
\hline & & & & & & \\
\hline & TRT & $16.1 \pm 0.5$ & $16.0 \pm 0.6$ & $16.2 \pm 0.6$ & & \\
\hline \multirow[t]{3}{*}{ Hematocrit (\%) } & PLA & $47.7 \pm 3.0$ & $48.5 \pm 3.3$ & $48.6 \pm 2.7$ & \multirow{3}{*}{$37.5-51.0$} & \multirow{3}{*}{0.35} \\
\hline & & & & & & \\
\hline & TRT & $47.3 \pm 1.5$ & $47.5 \pm 1.3$ & $47.9 \pm 1.6$ & & \\
\hline \multirow[t]{3}{*}{$\mathrm{MCV}(\mathrm{fL})$} & PLA & $89.7 \pm 3.1$ & $89.7 \pm 2.7$ & $89.6 \pm 2.7$ & \multirow{3}{*}{$79-97$} & \multirow{3}{*}{0.69} \\
\hline & & & & & & \\
\hline & TRT & $90.1 \pm 4.8$ & $90.6 \pm 3.9$ & $90.1 \pm 4.6$ & & \\
\hline \multirow[t]{3}{*}{$\mathrm{MCH}(\mathrm{pg})$} & PLA & $30.5 \pm 1.1$ & $30.3 \pm 1.0$ & $30.2 \pm 0.9$ & \multirow{3}{*}{$26.6-33.0$} & \multirow{3}{*}{0.88} \\
\hline & & & & & & \\
\hline & TRT & $30.7 \pm 1.4$ & $30.6 \pm 1.4$ & $30.5 \pm 1.4$ & & \\
\hline \multirow[t]{3}{*}{$\mathrm{MCHC}(\mathrm{g} / \mathrm{dL})$} & PLA & $34.0 \pm 1.0$ & $33.8 \pm 0.6$ & $33.7 \pm 0.7$ & \multirow{3}{*}{$31.5-35.7$} & \multirow{3}{*}{0.82} \\
\hline & & & & & & \\
\hline & TRT & $34.1 \pm 0.8$ & $33.8 \pm 0.6$ & $33.8 \pm 0.8$ & & \\
\hline \multirow[t]{3}{*}{ RDW (\%) } & PLA & $13.6 \pm 0.5$ & $13.4 \pm 0.4$ & $13.4 \pm 0.4$ & \multirow{3}{*}{$12.3-15.4$} & \multirow{3}{*}{0.09} \\
\hline & & & & & & \\
\hline & TRT & $13.2 \pm 0.6$ & $13.3 \pm 0.6$ & $13.2 \pm 0.6$ & & \\
\hline \multirow[t]{2}{*}{ Platelets } & PLA & $236.7 \pm 29.1$ & $239.4 \pm 45.4$ & $241.6 \pm 31.6$ & \multirow{3}{*}{$155-379$} & \multirow{3}{*}{0.37} \\
\hline & & & & & & \\
\hline (x10E3/uL) & TRT & $246.6 \pm 40.9$ & $251.9 \pm 39.5$ & $232.1 \pm 35.5$ & & \\
\hline \multirow[t]{3}{*}{ Neutrophils (\%) } & PLA & $53.5 \pm 8.3$ & $49.0 \pm 6.7$ & $49.5 \pm 9.1$ & \multirow{3}{*}{$40-74$} & \multirow{3}{*}{0.35} \\
\hline & & & & & & \\
\hline & TRT & $50.9 \pm 10.1$ & $51.0 \pm 7.7$ & $50.3 \pm 8.6$ & & \\
\hline Lymphs (\%) & PLA & $35.1 \pm 6.9$ & $38.9 \pm 6.5$ & $38.2 \pm 8.3$ & $14-46$ & 0.27 \\
\hline
\end{tabular}




\begin{tabular}{|c|c|c|c|c|c|c|}
\hline & TRT & $36.4 \pm 9.1$ & $36.7 \pm 5.9$ & $37.3 \pm 7.4$ & & \\
\hline \multirow[t]{3}{*}{ Monocytes (\%) } & PLA & $8.7 \pm 1.8$ & $9.4 \pm 2.1$ & $9.4 \pm 2.4$ & \multirow{3}{*}{$4-12$} & \multirow{3}{*}{0.63} \\
\hline & & & & & & \\
\hline & TRT & $9.4 \pm 2.4$ & $9.5 \pm 2.1$ & $9.1 \pm 1.6$ & & \\
\hline \multirow[t]{3}{*}{ Eos (\%) } & PLA & $2.1 \pm 1.2$ & $2.4 \pm 2.4$ & $2.5 \pm 2.3$ & \multirow{3}{*}{$0-5$} & \multirow{3}{*}{0.13} \\
\hline & & & & & & \\
\hline & TRT & $2.7 \pm 2.2$ & $2.3 \pm 1.9$ & $2.9 \pm 2.3$ & & \\
\hline \multirow[t]{3}{*}{ Basos (\%) } & PLA & $0.6 \pm 0.7$ & $0.4 \pm 0.7$ & $0.4 \pm 0.7$ & \multirow{3}{*}{$0-3$} & \multirow{3}{*}{0.5} \\
\hline & & & & & & \\
\hline & TRT & $0.6 \pm 0.5$ & $0.5 \pm 0.5$ & $0.6 \pm 0.5$ & & \\
\hline Neutrophils & PLA & $3.1 \pm 1.4$ & $2.8 \pm 0.8$ & $2.9 \pm 1.0$ & \multirow{3}{*}{$1.4-7.0$} & \multirow{3}{*}{0.60} \\
\hline (Absolute) & & & & & & \\
\hline (x10E3/uL) & TRT & $3.1 \pm 1.4$ & $3.0 \pm 1.2$ & $3.1 \pm 0.9$ & & \\
\hline Lymphs (Absolute) & PLA & $1.9 \pm 0.1$ & $2.2 \pm 0.3$ & $2.1 \pm 0.4$ & \multirow[b]{2}{*}{$0.7-3.1$} & \multirow[b]{2}{*}{0.21} \\
\hline (x10E3/uL) & TRT & $2.0 \pm 0.5$ & $2.1 \pm 0.5$ & $2.2 \pm 0.6$ & & \\
\hline Monocytes & PLA & $0.5 \pm 0.2$ & $0.5 \pm 0.1$ & $0.5 \pm 0.2$ & \multirow{3}{*}{$0.1-0.9$} & \multirow{3}{*}{0.76} \\
\hline (Absolute) & & & & & & \\
\hline (x10E3/uL) & TRT & $0.5 \pm 0.2$ & $0.6 \pm 0.2$ & $0.5 \pm 0.2$ & & \\
\hline Eos (Absolute) & PLA & $0.1 \pm 0.1$ & $0.1 \pm 0.1$ & $0.1 \pm 0.1$ & \multirow[b]{2}{*}{$0.0-0.4$} & \multirow[b]{2}{*}{0.51} \\
\hline (x10E3/uL) & TRT & $0.2 \pm 0.1$ & $0.2 \pm 0.1$ & $0.2 \pm 0.1$ & & \\
\hline Baso (Absolute) & PLA & $0.0 \pm 0.0$ & $0.0 \pm 0.0$ & $0.0 \pm 0.0$ & \multirow[b]{2}{*}{$0.0-0.2$} & \multirow[b]{2}{*}{0.21} \\
\hline (x10E3/uL) & TRT & $0.0 \pm 0.0$ & $0.0 \pm 0.0$ & $0.0 \pm 0.0$ & & \\
\hline
\end{tabular}

1 Data are presented as mean \pm standard deviation. The $p$-value is derived from an ANOVA and

2 representative of a group by time effect. 
Table 4. Blood Chemistry Data.

\begin{tabular}{|c|c|c|c|c|c|c|}
\hline Variable & Treatment & PRE & Week 8 & POST & $\begin{array}{l}\text { Reference } \\
\text { Interval }\end{array}$ & $\mathrm{p}$ \\
\hline \multirow{2}{*}{$\begin{array}{l}\text { Serum Glucose } \\
(\mathrm{mg} / \mathrm{dL})\end{array}$} & PLA & $90.5 \pm 11.0$ & $89.3 \pm 3.6$ & $90.2 \pm 5.4$ & \multirow{2}{*}{$65-99$} & \multirow{2}{*}{0.91} \\
\hline & TRT & $87.9 \pm 8.8$ & $87.9 \pm 6.0$ & $86.6 \pm 6.5$ & & \\
\hline \multirow[t]{2}{*}{ BUN (mg/dL) } & PLA & $17.3 \pm 4.9$ & $17.8 \pm 3.9$ & $17.5 \pm 4.2$ & \multirow{2}{*}{$6-20$} & \multirow{2}{*}{0.94} \\
\hline & TRT & $18.6 \pm 6.3$ & $18.7 \pm 4.1$ & $17.8 \pm 4.1$ & & \\
\hline \multirow{2}{*}{$\begin{array}{l}\text { Serum Creatinine } \\
(\mathrm{mg} / \mathrm{dL})\end{array}$} & PLA & $0.97 \pm 0.12$ & $1.04 \pm 0.12^{\mathrm{a}}$ & $1.13 \pm 0.17^{* a b}$ & \multirow{2}{*}{$0.76-1.27$} & \multirow{2}{*}{0.01} \\
\hline & TRT & $0.98 \pm 0.09$ & $1.04 \pm 0.09^{\mathrm{a}}$ & $1.05 \pm 0.12^{\mathrm{a}}$ & & \\
\hline \multirow[t]{2}{*}{ eGFR (mL/min/1.73) } & PLA & $105.6 \pm 12.9$ & $98.7 \pm 12.5$ & $91.0 \pm 14.9$ & \multirow{2}{*}{$>59$} & \multirow{2}{*}{0.06} \\
\hline & TRT & $105.3 \pm 10.4$ & $99.5 \pm 10.6$ & $98.1 \pm 12.5$ & & \\
\hline \multirow{2}{*}{$\begin{array}{l}\text { BUN/Creatinine } \\
\text { Ratio }\end{array}$} & PLA & $18.0 \pm 5.9$ & $17.3 \pm 4.0$ & $15.8 \pm 4.4$ & \multirow{2}{*}{$8-19$} & \multirow{2}{*}{0.63} \\
\hline & TRT & $18.7 \pm 5.4$ & $18.1 \pm 3.9$ & $17.0 \pm 3.3$ & & \\
\hline \multirow{2}{*}{$\begin{array}{l}\text { Serum Sodium } \\
\text { (mmol/L) }\end{array}$} & PLA & $138.8 \pm 1.8$ & $139.6 \pm 1.3$ & $140.4 \pm 2.1$ & \multirow{2}{*}{$134-144$} & \multirow{2}{*}{0.75} \\
\hline & TRT & $139.1 \pm 1.0$ & $139.1 \pm 1.7$ & $140.4 \pm 1.9$ & & \\
\hline \multirow{2}{*}{$\begin{array}{l}\text { Serum Potassium } \\
\text { (mmol/L) }\end{array}$} & PLA & $4.4 \pm 0.4$ & $4.5 \pm 0.5$ & $4.3 \pm 0.3$ & \multirow{2}{*}{$3.5-5.2$} & \multirow{2}{*}{0.10} \\
\hline & TRT & $4.3 \pm 0.3$ & $4.5 \pm 0.2$ & $4.5 \pm 0.3$ & & \\
\hline \multirow{2}{*}{$\begin{array}{l}\text { Serum Chloride } \\
\text { (mmol/L) }\end{array}$} & PLA & $102.1 \pm 1.6$ & $101.0 \pm 1.4$ & $102.4 \pm 2.2$ & \multirow{2}{*}{$97-108$} & \multirow{2}{*}{0.94} \\
\hline & TRT & $101.1 \pm 1.7$ & $100.2 \pm 1.9$ & $101.1 \pm 1.9$ & & \\
\hline \multirow{2}{*}{$\begin{array}{l}\text { Carbon Dioxide } \\
(\mathrm{mmol} / \mathrm{L})\end{array}$} & PLA & $23.6 \pm 2.4$ & $22.1 \pm 1.5$ & $22.5 \pm 1.5$ & \multirow{2}{*}{$19-28$} & \multirow{2}{*}{0.36} \\
\hline & TRT & $23.9 \pm 1.8$ & $22.6 \pm 1.2$ & $24.1 \pm 1.2$ & & \\
\hline Serum Calcium & PLA & $9.4 \pm 0.4$ & $9.4 \pm 0.3$ & $9.3 \pm 0.3$ & 2-10 & 044 \\
\hline & TRT & $9.5 \pm 0.4$ & $9.4 \pm 0.3$ & $9.5 \pm 0.2$ & 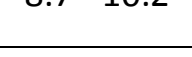 & \\
\hline Serum Protein $(\mathrm{g} / \mathrm{dL})$ & PLA & $7.1 \pm 0.3$ & $6.9 \pm 0.3$ & $6.9 \pm 0.3$ & $60-85$ & 082 \\
\hline & TRT & $7.0 \pm 0.2$ & $6.8 \pm 0.3$ & $6.9 \pm 0.3$ & & \\
\hline Serum Albumin & PLA & $4.4 \pm 0.3$ & $4.6 \pm 0.2$ & $4.6 \pm 0.2$ & $35-55$ & 076 \\
\hline$(g / d L)$ & TRT & $4.5 \pm 0.2$ & $4.6 \pm 0.2$ & $4.7 \pm 0.3$ & $3.5-5.5$ & 0.16 \\
\hline Globulin (g/dL) & PLA & $2.6 \pm 0.2$ & $2.3 \pm 0.2$ & $2.3 \pm 0.2$ & $15-45$ & 093 \\
\hline & TRT & $2.6 \pm 0.2$ & $2.2 \pm 0.2$ & $2.2 \pm 0.3$ & $1.5-4 . J$ & 0.5 \\
\hline Albumin:Globulin & PLA & $1.7 \pm 0.2$ & $2.0 \pm 0.2$ & $2.0 \pm 0.3$ & $11-25$ & 084 \\
\hline Ratio & TRT & $1.8 \pm 0.2$ & $2.1 \pm 0.3$ & $2.2 \pm 0.3$ & $1.1-2.0$ & \\
\hline Bilirubin (mg/dl) & PLA & $0.6 \pm 0.2$ & $0.6 \pm 0.2$ & $0.6 \pm 0.2$ & $00-12$ & 0.35 \\
\hline & TRT & $0.7 \pm 0.3$ & $0.7 \pm 0.4$ & $0.6 \pm 0.2$ & & \\
\hline Alkaline Phosphatase & PLA & $76.4 \pm 13.8$ & $81.3 \pm 14.6$ & $79.9 \pm 16.1$ & $39-117$ & 0.40 \\
\hline (IU/L) & TRT & $73.4 \pm 18.4$ & $83.4 \pm 23.0$ & $84.5 \pm 24.2$ & $39-11 /$ & 0.40 \\
\hline AST (IU/L) & PLA & $25.5 \pm 7.8$ & $26.9 \pm 7.4$ & $25.8 \pm 8.9$ & $0-40$ & 006 \\
\hline & TRT & $32.9 \pm 13.1$ & $27.5 \pm 8.5$ & $24.6 \pm 5.9$ & $0-40$ & 0.00 \\
\hline ALT (IU/L) & PLA & $23.7 \pm 4.9$ & $24.3 \pm 7.0$ & $22.5 \pm 6.3$ & $0-44$ & 0.61 \\
\hline & TRT & $27.6 \pm 12.7$ & $25.1 \pm 7.3$ & $25.1 \pm 6.0$ & & \\
\hline
\end{tabular}


1 Data are presented as mean \pm standard deviation. * indicates significantly different from PLA at the

2 corresponding time point. The p-value is derived from an ANOVA and representative of a main effect for

3 group by time. Significant time effects are indicated by a (different from pre) and b (different from week

$48)$. 


\section{Figure Legend}

2 Figure 1. Changes in CSA. Delta values between corresponding weeks are presented as mean \pm standard

3 deviation. * indicates significantly different from PLA. Significance was determined by Independent T-

4 tests. + indicates a significant within-group difference.

5 Figure 2. Changes in MT. Delta values between corresponding weeks are presented as mean \pm standard

6 deviation. * indicates significantly different from PLA. Significance was determined by Independent T-

7 tests. + indicates a significant within-group difference. 


\section{Changes in CSA}

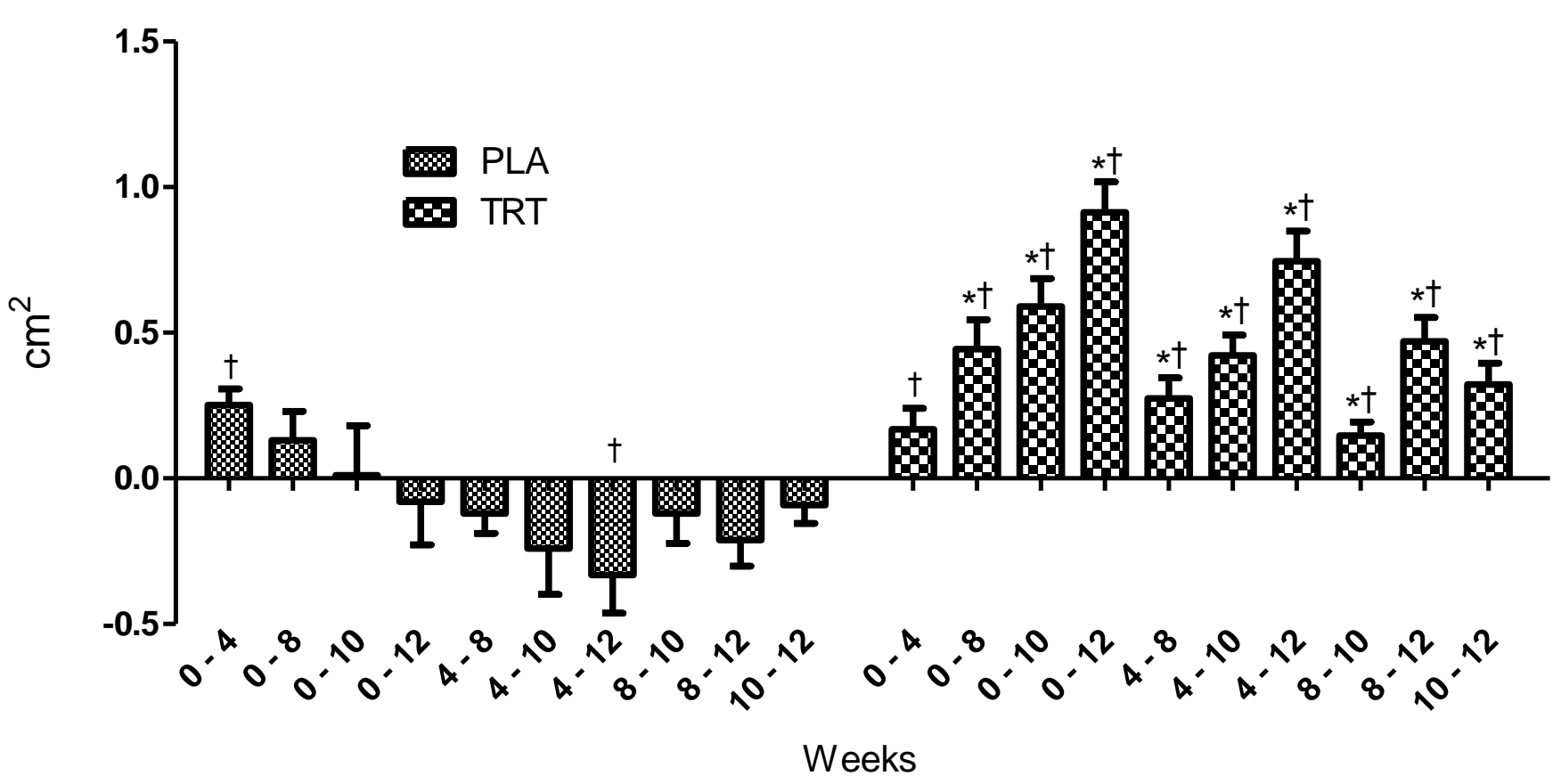




\section{Changes in Muscle Thickness}

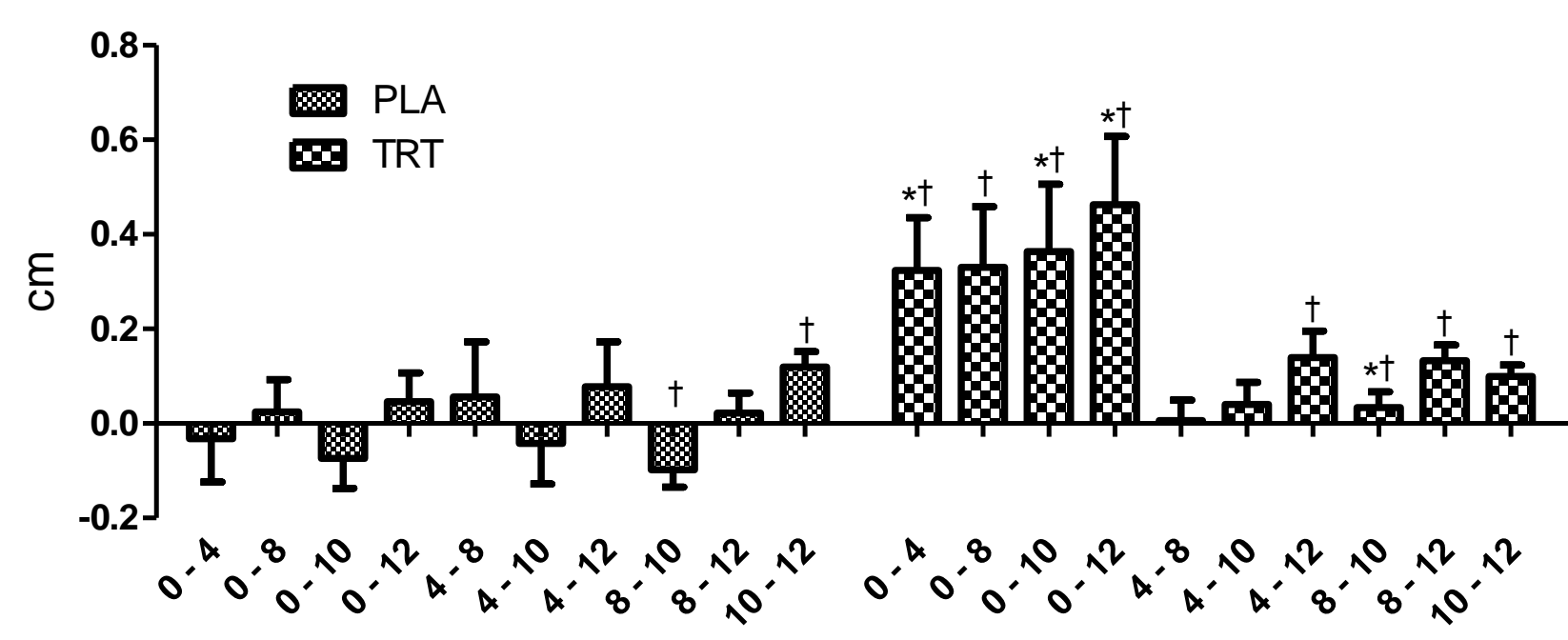

Weeks 